\title{
Effect of Axial Deformation on Elastic Properties of Irregular Honeycomb Structure
}

Ning Wang and Qingtian Deng*

\begin{abstract}
Irregular honeycomb structures occur abundantly in nature and in man-made products, and are an active area of research. In this paper, according to the optimization of regular honeycomb structures, two types of irregular honeycomb structures with both positive and negative Poisson's ratios are presented. The elastic properties of irregular honeycombs with varying structure angles were investigated through a combination of material mechanics and structural mechanics methods, in which the axial deformation of the rods was considered. The numerical results show that axial deformation has a significant influence on the elastic properties of irregular honeycomb structures. The elastic properties of the structure can be considered by the enclosed area of the unit structure, the shape of the unit structure, and the elastic properties of the original materials. The elastic properties considering the axial deformation of rods studied in this study can provide a reference for other scholars.
\end{abstract}

Keywords: Axial deformation, Elastic modulus, Positive Poisson's ratio, Negative Poisson's ratio, Irregular honeycombs

\section{Introduction}

Honeycomb structures have many excellent characteristics, such as a higher elastic modulus [1], higher shear modulus [2], and higher energy absorption [3-6]. Therefore, negative Poisson's ratio materials are widely used in aerospace $[7,8]$, automobiles, ships $[9,10]$, and other fields. Zhang et al. [11] studied a hierarchical regular hexagonal honeycomb structure. Mukhopadhyay and Adhikari [12] found that the elastic modulus of irregular honeycombs was highly influenced by the structural irregularity in auxetic honeycombs. Yang and Deng [13] reviewed the development of materials and structures with a negative Poisson's ratio and the prospect development of porous materials. Lan et al. [14] analyzed a thin-walled honeycomb structure and investigated the effects of its structural and material parameters. Upreti et al. [15] studied honeycomb sandwich composites with a hexagonal honeycomb core and found that deformation decreased with increasing face sheet thickness. Thus,

*Correspondence: dengqt@chd.edu.cn

School of Science, Chang'an University, Xi'an 710064, China honeycomb structures have always been of profound interest to the research community.

Compared with traditional regular structures, irregular structures are more widely used in engineering applications owing to their better compressibility $[16,17]$ and higher buckling $[18,19]$. Other important research areas related to the study of different honeycomb structures are their thermal and acoustic properties [20-23]. Therefore, much research has been carried out to predict the elastic properties of irregular honeycombs [24, 25]. Deng and Yang [26] investigated the behaviors of functionally graded structures in three different types of elastic moduli. In the numerical results, it was found that Poisson's ratio exhibits appreciable effects on bearing capacity, which indicates that static properties can be improved by optimal design of cell shape material distribution and computational methodology. $\mathrm{Hu}$ et al. [27] studied the Poisson's ratio of re-entrant honeycombs using numerical and structural methods. Huang et al. [28] presented a novel zero in-plane Poisson's ratio honeycomb designed for large out-of-plane deformations and studied the relationship between in-plane stiffness and geometric parameters. Wang et al. [29] analyzed the 
elastic modulus and Poisson's ratio of the re-entrant auxetic cellular structure in two principal directions. Bubert et al. [30] fabricated a skin supported by an accordion honeycomb and analyzed the in-plane equivalent elastic moduli in two directions without discussing the modulus in the third direction. Li et al. [31] studied the dynamic crushing response of irregular honeycomb structures and found that the propagation velocity of the stress wave is different in different honeycomb structures. Liu et al. [32] analyzed the effect of Poisson's ratios on the crashworthiness of in-plane honeycombs. Therefore, honeycomb structures [33], especially irregular structures [34], have wide research prospects.

In this study, the equivalent elastic modulus and Poisson's ratio of an irregular honeycomb in the $\sigma_{1}$ and $\sigma_{2}$ directions are derived using Castigliano's second theorem, and the internal bending moment, axial deformation, and Poisson's ratio of the original material are considered. Finally, the results in this paper are verified with the results reported in Ref. [24], and the effects of different structural shapes and axial deformation of rods on the elastic properties are analyzed. The results show that when considering the axial deformation, the absolute value of Poisson's ratio is lower than that without considering the axial deformation, and the variation in the structural shape has a slight influence on the elastic properties.

\section{Irregular Honeycomb Structure}

Figure 1 shows two types of regular structures: (a) and (b) are regular honeycomb structures with both positive and negative Poisson's ratios. Figure 2 shows two types of irregular structures: (a) and (b) are irregular honeycomb structures with both positive and negative Poisson's ratios.

\section{Irregular Honeycomb Structures with Positive Poisson's Ratio}

Figure 3 shows irregular honeycomb structures loaded in two different directions, where $\sigma_{1}$ and $\sigma_{2}$ are uniformly distributed loads in two mutually perpendicular directions.

\subsection{Elastic Modulus in the $\sigma_{1}$ Direction}

Figure 4 shows the structural parameters and force analysis of $\operatorname{rod} A B$, where $b$ is the thickness of the unit structure, $t$ is the depth of the unit structure, $l_{i}$ and $h$ are the lengths of the inclined cell walls with inclination angle $\theta$ and the length of the vertical rod, respectively. The moment $M_{1}$ of $\operatorname{rod} A B$ can be expressed as

$$
M_{1}=\frac{P_{1} l_{1} \sin \theta_{1}}{2},
$$

where $P_{1}=\sigma\left(h+l_{1} \sin \theta_{1}\right) b$ is the force in the $\sigma_{1}$ direction.

From the standard beam theory [33], the deflection $\delta_{A B}$ of $\operatorname{rod} A B$ can be expressed as

$$
\delta_{A B}=\frac{p_{1} l_{1}^{3} \sin \theta_{1}}{12 E I} .
$$

Axial force $F^{\prime}$ along $\operatorname{rod} A B$ is

$$
F^{\prime}=p_{1} \cos \theta_{1} .
$$

Axial deformation $\Delta l$ can be expressed as

$$
\Delta l=\frac{F^{\prime} L}{E A}=\frac{p_{1} \cos \theta_{1} l_{1}}{E b t},
$$

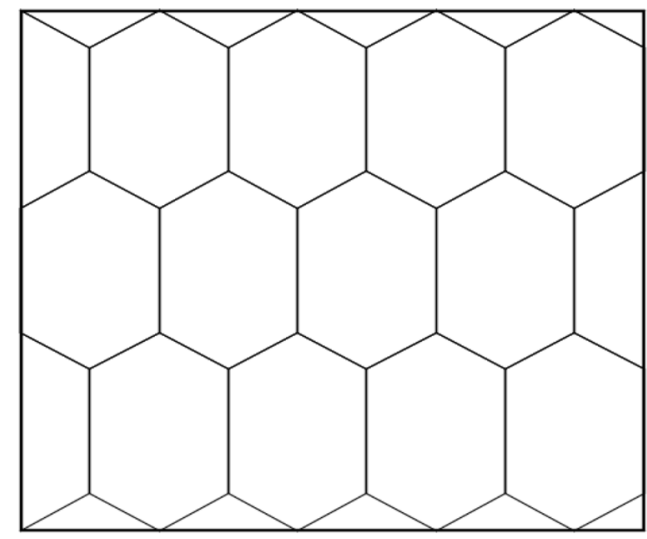

a Regular honeycomb structure

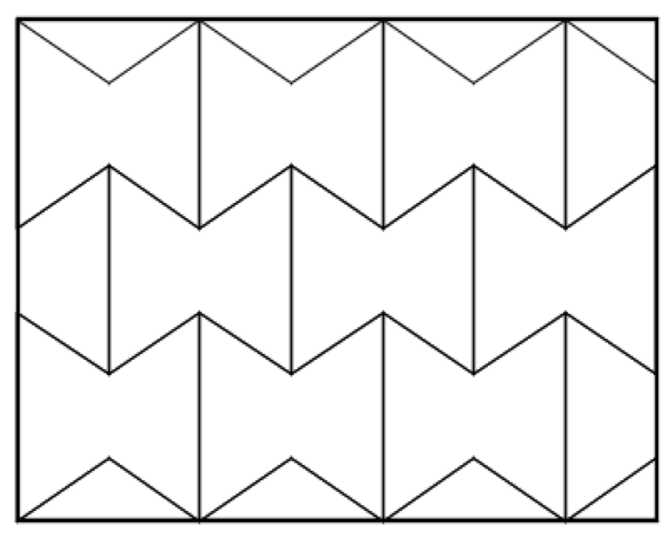

b Regular negative Poisson's ratio structure

Figure 1 Regular structures 


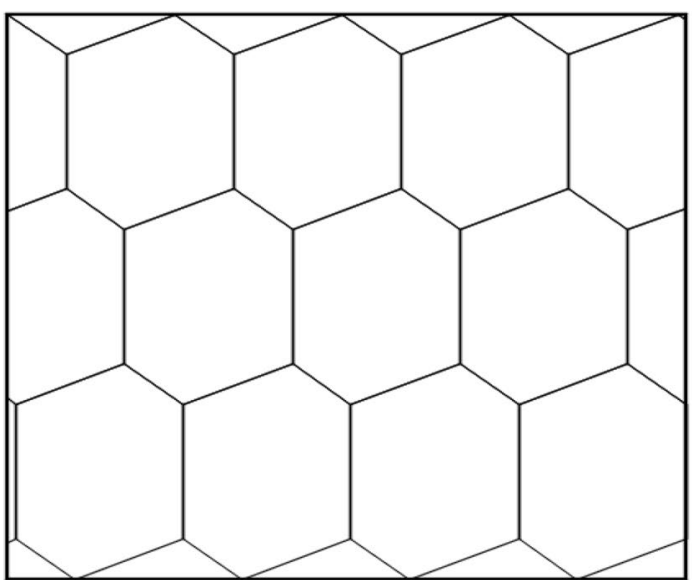

a Irregular honeycomb structure

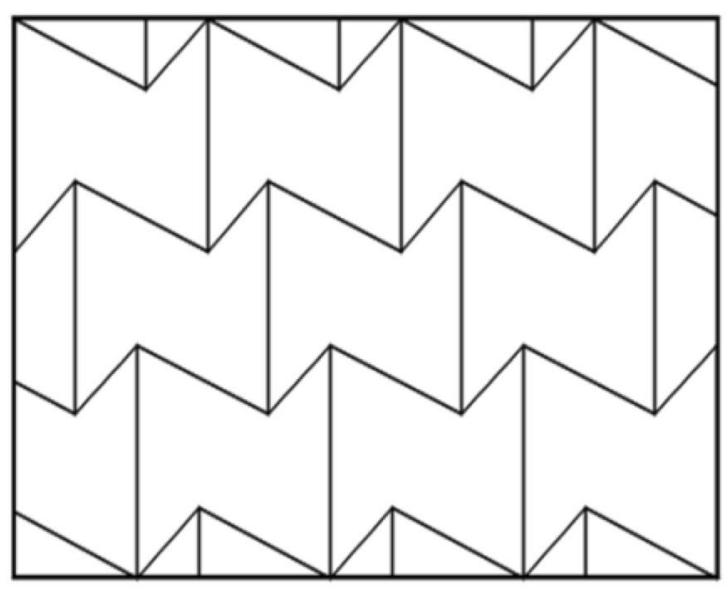

b Irregular negative Poisson's ratio structure
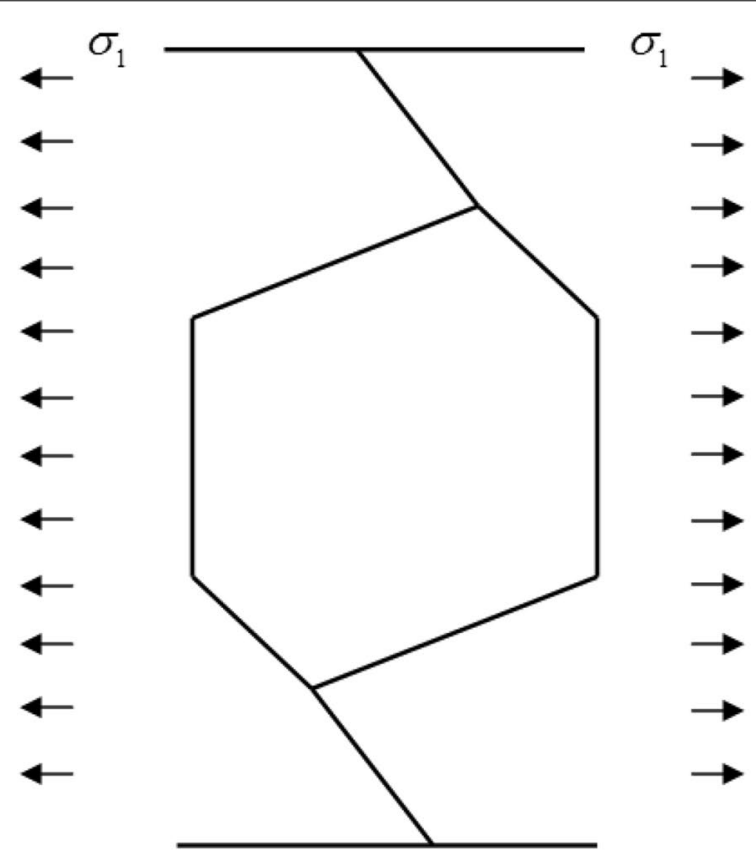

a Stress along the $\sigma_{1}$ direction

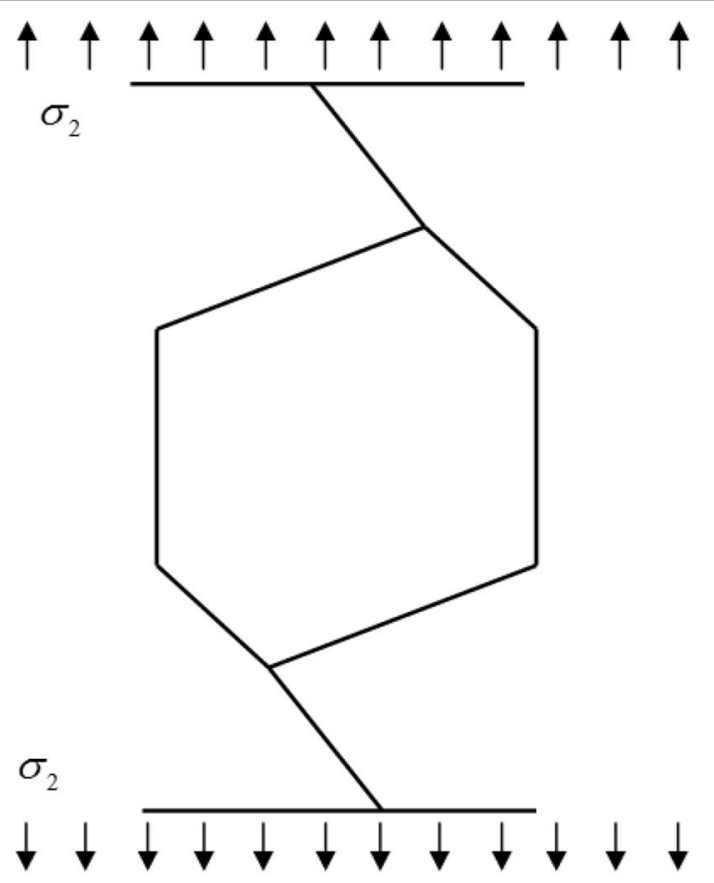

b Stress along the $\sigma_{2}$ direction

Figure 3 Applied tensile stresses in the $(\mathbf{a}) \sigma_{1}$ and (b) $\sigma_{2}$ directions

where $E$ is the elastic moduli of the original materials, and the total deformation $\delta_{1}$ of $\operatorname{rod} A B$ along the $\sigma_{1}$ direction is

$$
\delta_{1}=\delta_{A B} \sin \theta_{1}+\Delta l \cos \theta_{1}=\frac{p_{1} l_{1}^{3} \sin ^{2} \theta_{1}}{12 E I}+\frac{p_{1} \cos ^{2} \theta_{1} l_{1}}{E b t} .
$$

Similarly, the total deformation $\delta_{2}$ of $\operatorname{rod} B C$ along the $\sigma_{1}$ direction can be expressed as follows:

$$
\delta_{2}=\delta_{B C} \sin \theta_{2}+\Delta l \cos \theta_{2}=\frac{p_{1} l_{2}^{3} \sin ^{2} \theta_{2}}{12 E I}+\frac{p_{1} \cos ^{2} \theta_{2} l_{2}}{E b t} .
$$




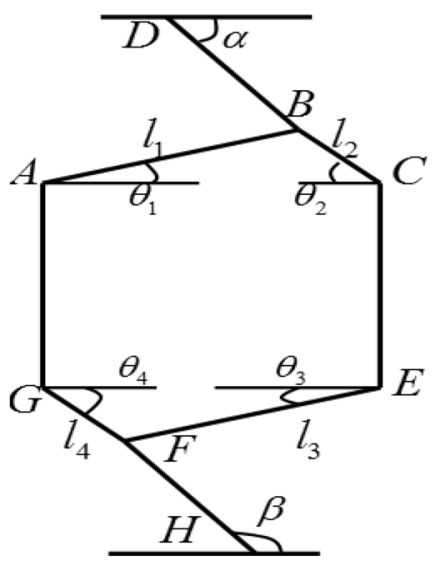

a Structure parameters

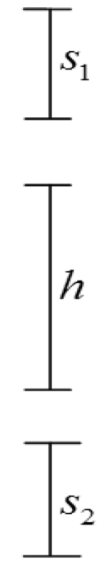

Figure 4 Irregular structure diagram and force analysis of rod $A B$

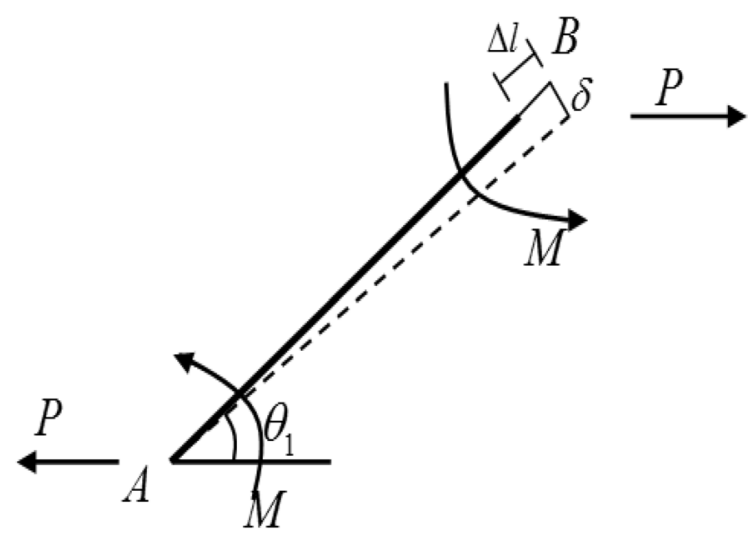

b Force analysis of $\operatorname{rod} A B$
Combing Eqs. (2)-(6), the strain $\varepsilon_{1}$ parallel to the $\sigma_{1}$ direction is given by

$$
\varepsilon_{1}=\frac{\delta_{1}+\delta_{2}}{l_{1} \cos \theta_{1}+l_{2} \cos \theta_{2}}=\frac{\sigma \gamma}{l_{1} \cos \theta_{1}+l_{2} \cos \theta_{2}},
$$

where

$$
\begin{aligned}
\gamma= & b\left[\left(h+l_{1} \sin \theta_{1}\right)\left(\frac{l_{1}^{3} \sin ^{2} \theta_{1}}{12 E I}+\frac{\cos ^{2} \theta_{1} l_{1}}{E b t}\right)\right. \\
& \left.+\left(h+l_{2} \sin \theta_{2}\right)\left(\frac{l_{2}^{3} \sin ^{2} \theta_{2}}{12 E I}+\frac{\cos ^{2} \theta_{2} l_{2}}{E b t}\right)\right] .
\end{aligned}
$$

Thus, the elastic modulus $E_{1 U}$ in the $\sigma_{1}$ direction can be expressed as follows:

$$
E_{1 U}=\frac{\left(l_{1} \cos \theta_{1}+l_{2} \cos \theta_{2}\right)}{\gamma} .
$$

\subsection{Elastic Modulus in the $\sigma_{2}$ Direction}

To derive the expression of the transverse elastic modulus, stress $\sigma_{2}$ is applied, as shown in Figure 3(b). Figure 5 shows that the deflection of rod $B D$ consists of two parts: bending deformation and rotational deformation. The bending deformation $\delta_{2 v b}$ caused by the moment $M_{1}$ in the $\sigma_{2}$ direction can be expressed as

$$
\delta_{2 v b}=\left(\frac{w \cos \alpha\left(\frac{s_{1}}{\sin \alpha}\right)^{3}}{3 E I}\right) \cos \alpha,
$$

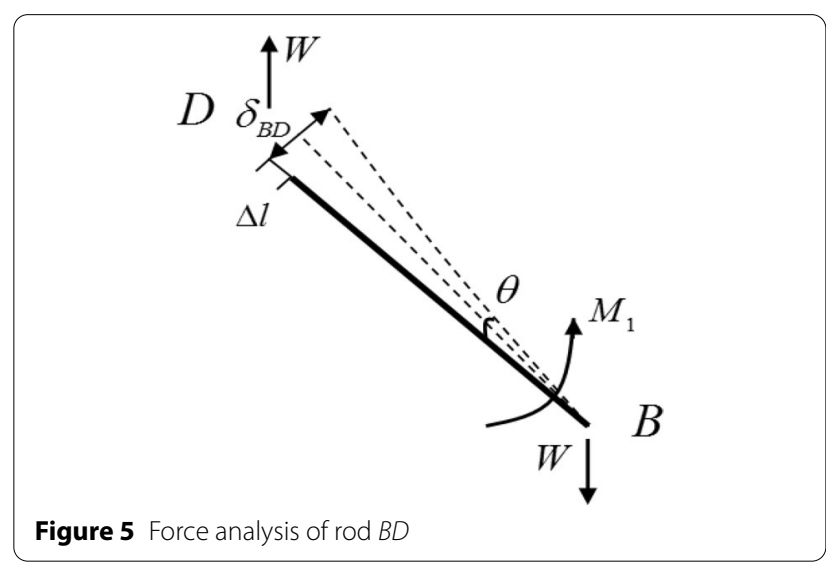

where

$$
\left\{\begin{array}{l}
w=\sigma_{2}\left(l_{1} \cos \theta_{1}+l_{2} \cos \theta_{2}\right) b \\
I=b t^{3} / 12 \\
M_{1}=w s_{1} \cot \alpha
\end{array}\right.
$$

Because the rotation angles of the three rods connected to point $B$ are identical, the rotation angle $\phi$ of joint $B$ can be written as

$$
\phi=\frac{M_{1} l_{1}}{l_{1}+l_{2}} \frac{l_{1}}{6 E I} .
$$

Thus, the deformation $\delta_{2 v r}$ of the cell wall with an inclination angle $\alpha$ in the $\sigma_{2}$ direction is given by: 


$$
\delta_{2 v r}=\phi\left(\frac{s_{1}}{\sin \alpha}\right) \cos \alpha
$$

Therefore, the total deformations of $\operatorname{rod} B D$ and $\operatorname{rod} F H$ in the $\sigma_{2}$ direction are

$$
\begin{aligned}
\delta_{2 B D}= & \left(\frac{w \cos \alpha\left(\frac{s_{1}}{\sin \alpha}\right)^{3}}{3 E I}\right) \cos \alpha+\frac{M_{1} l_{1}}{l_{1}+l_{2}} \frac{l_{1}}{6 E I}\left(\frac{s_{1}}{\sin \alpha}\right) \cos \alpha \\
\delta_{v 2 F H}= & \left(\frac{w \cos \beta\left(\frac{s_{2}}{\sin \beta}\right)^{3}}{3 E I}\right) \cos \beta \\
& +\frac{M_{1} l_{4}}{l_{3}+l_{4}} \frac{l_{4}}{6 E I}\left(\frac{s_{2}}{\sin \beta}\right) \cos \beta .
\end{aligned}
$$

Now, the axial deformations of $\operatorname{rod} B D$ and $\operatorname{rod} F H$ in the $\sigma_{2}$ direction can be expressed as

$$
\begin{aligned}
& \Delta l_{2 B D}=\frac{w s_{1}}{E b t} \sin \alpha, \\
& \Delta l_{2 F H}=\frac{w s_{2}}{E b t} \sin \beta .
\end{aligned}
$$

The deflections $\delta_{\nu B D}$ and $\delta_{\nu G F}$ of rods $A B$ and GF (Figure 6) in the $\sigma_{2}$ direction can be expressed as

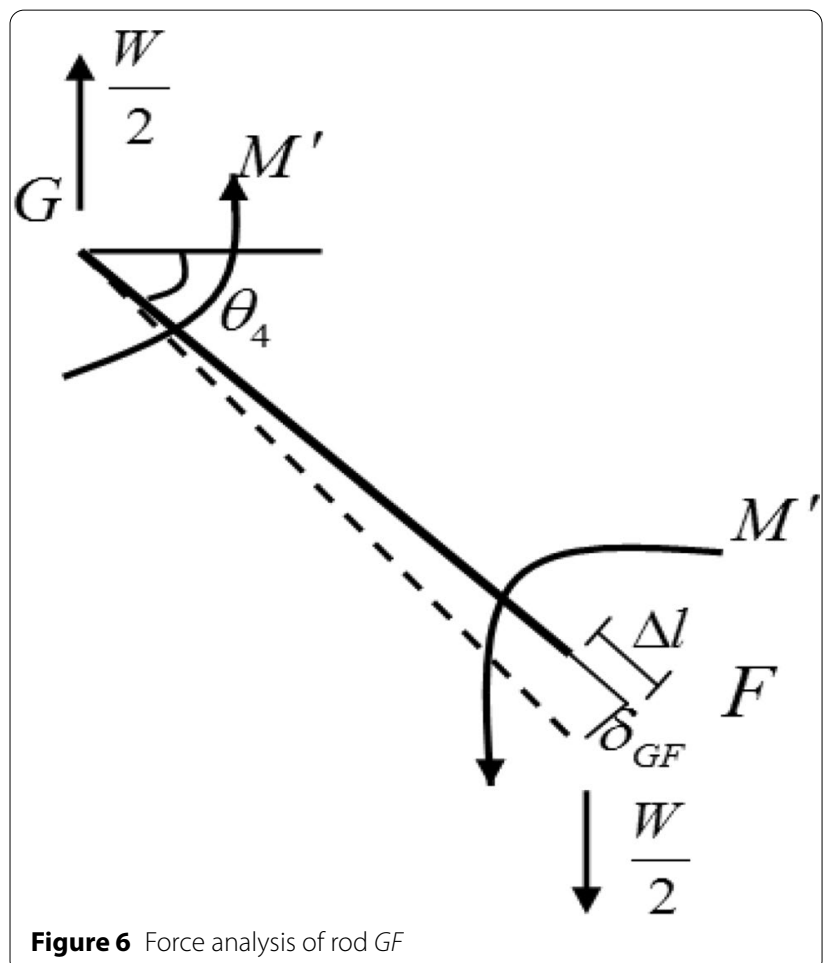

$$
\begin{aligned}
& \delta_{v A B}=\frac{\left(\frac{l_{1} w}{l_{1}+l_{2}} \cos \theta_{1}\right) l_{1}^{3}}{12 E I} \cos \theta_{1}, \\
& \delta_{v G F}=\frac{\left(\frac{l_{4} w}{l_{3}+l_{4}} \cos \theta_{4}\right) l_{4}^{3}}{12 E I} \cos \theta_{4} .
\end{aligned}
$$

The axial deformation of rods $A B$ and $G F$ in the $\sigma_{2}$ direction can be expressed as

$$
\begin{aligned}
& \Delta l_{2}^{\prime}=\frac{\frac{l_{1} w}{l_{1}+l_{2}} \sin \theta_{1} l_{1}}{E b t} \sin \theta_{1}, \\
& \Delta l_{3}^{\prime}=\frac{\frac{l_{4} w}{l_{3}+l_{4}} \sin \theta_{4} l_{4}}{E b t} \sin \theta_{4} .
\end{aligned}
$$

Thus, the total deformation $\delta_{2}$ of the structure in the $\sigma_{2}$ direction can be expressed as

$$
\begin{aligned}
\delta_{2}= & \delta_{v 2 B D}+\delta_{v 2 F H}+\Delta l_{2 B D}+\Delta l_{2 F H} \\
& +\delta_{v A B}+\delta_{v G F}+\Delta l_{2}^{\prime}+\Delta l_{3}^{\prime},
\end{aligned}
$$

while Eq. (22) can be rewritten as follows:

$$
\delta_{2}=w \varphi
$$

where

$$
\begin{aligned}
\varphi= & {\left[\left(\frac{\cos \alpha\left(\frac{s_{1}}{\sin \alpha}\right)^{3}}{3 E I}\right) \cos \alpha+\frac{\mathrm{s}_{1} \cot \alpha l_{1}}{l_{1}+l_{2}} \frac{l_{1}}{6 E I}\left(\frac{s_{1}}{\sin \alpha}\right) \cos \alpha\right.} \\
& +\left(\frac{\cos \beta\left(\frac{s_{2}}{\sin \beta}\right)^{3}}{3 E I}\right) \cos \beta+\frac{\mathrm{s}_{1} \cot \alpha l_{4}}{l_{3}+l_{4}} \frac{l_{4}}{6 E I}\left(\frac{s_{2}}{\sin \beta}\right) \cos \beta \\
& +\frac{s_{1}}{E b t} \sin \alpha+\frac{s_{2}}{E b t} \sin \beta+\frac{\left(\frac{l_{1}}{l_{1}+l_{2}} \cos \theta_{1}\right) l_{1}^{3}}{12 E I} \cos \theta_{1} \\
& +\frac{\left(\frac{l_{4}}{l_{3}+l_{4}} \cos \theta_{4}\right) l_{4}^{3}}{12 E I} \cos \theta_{4}+\frac{\frac{l_{1}}{l_{1}+l_{2}} \sin \theta_{1} l_{1}}{E b t} \sin \theta_{1} \\
& \left.+\frac{\frac{l_{4}}{l_{+}+l_{4}} \sin \theta_{4} l_{4}}{E b t} \sin \theta_{4}\right]
\end{aligned}
$$

Strain $\varepsilon_{2}$ in the $\sigma_{2}$ direction can be obtained as

$$
\begin{aligned}
\varepsilon_{2} & =\frac{\delta_{2}}{h+s_{1}+s_{2}+l_{1} \sin \theta_{1}+l_{4} \sin \theta_{4}} \\
& =\frac{w \varphi}{h+s_{1}+s_{2}+l_{1} \sin \theta_{1}+l_{4} \sin \theta_{4}},
\end{aligned}
$$

where $w=\sigma_{2}\left(l_{1} \cos \theta_{1}+l_{2} \cos \theta_{2}\right) b$; thus, the elastic modulus in the $\sigma_{2}$ direction of the structure can be expressed as 


$$
E_{2 U}=\frac{\sigma_{2}}{\varepsilon_{2}}=\frac{h+s_{1}+s_{2}+l_{1} \sin \theta_{1}+l_{4} \sin \theta_{4}}{\left(l_{1} \cos \theta_{1}+l_{2} \cos \theta_{2}\right) b \varphi} .
$$

\subsection{Poisson's Ratio $v_{12}$}

Poisson's ratios were calculated by taking the negative ratios of strains normal to and parallel to the loading direction. Poisson's ratio $v_{12}$ of the unit structure can be defined as

$$
\nu_{12}=-\frac{\varepsilon_{2}}{\varepsilon_{1}},
$$

where $\varepsilon_{1}$ and $\varepsilon_{2}$ are strains in the $\sigma_{1}$ and $\sigma_{2}$ directions, respectively, due to the load in the $\sigma_{1}$ direction. In addition, $\varepsilon_{1}$ can be obtained from Eq. (7) and $\varepsilon_{2}$ can be expressed as

$$
\varepsilon_{2}=-\frac{\delta_{1}^{\prime}+\delta_{2}^{\prime}}{h+s_{1}+s_{2}+l_{1} \sin \theta_{1}+l_{4} \sin \theta_{4}},
$$

where

\subsection{Poisson's Ratio $v_{21}$}

Poisson's ratio of a structure for loading in the $\sigma_{2}$ direction can be expressed as

$$
v_{21}=-\frac{\varepsilon_{1}^{\prime}}{\varepsilon_{2}^{\prime}},
$$

where $\varepsilon_{1}^{\prime}$ and $\varepsilon_{2}^{\prime}$ are the strains in the $\sigma_{1}$ and $\sigma_{2}$ directions, respectively. $\varepsilon_{2}^{\prime}$ can be obtained from Eq. (23) as

$$
\varepsilon_{2}^{\prime}=\frac{w \varphi}{h+s_{1}+s_{2}+l_{1} \sin \theta_{1}+l_{4} \sin \theta_{4}},
$$

where $w=\sigma_{2}\left(l_{1} \cos \theta_{1}+l_{2} \cos \theta_{2}\right) b$, and $\varepsilon_{1}^{\prime}$ can be obtained as

$$
\varepsilon_{1}^{\prime}=-\frac{\delta_{v A B 1}+\delta_{v B C 1}}{l_{1} \cos \theta_{1}+l_{2} \cos \theta_{2}}=-\frac{w \varphi^{\prime}}{l_{1} \cos \theta_{1}+l_{2} \cos \theta_{2}},
$$

where $\delta_{v A B 1}$ and $\delta_{v B C 1}$ are the deformations in the $\sigma_{1}$ direction due to the load in the $\sigma_{2}$ direction

$$
\left\{\begin{array}{l}
\delta_{1}^{\prime}=\delta_{A B} \cos \theta_{1}+\Delta l \sin \theta_{1}=\frac{p_{1} l_{1}^{3} \sin \theta_{1} \cos \theta_{1}}{12 L I}+\frac{p_{1} l_{1} \sin \theta_{1} \cos \theta_{1}}{E b t} \\
\delta_{2}^{\prime}=\delta_{G F} \cos \theta_{4}+\Delta l \sin \theta_{4}=\frac{p_{2} l_{4}^{3} \sin \theta_{4} \cos \theta_{4}}{12 E I}+\frac{p_{2} l_{4} \sin \theta_{4} \cos \theta_{4}}{E b t}
\end{array} .\right.
$$

The Poisson's ratio of a structure in the $\sigma_{1}$ direction can be expressed as

$$
v_{12}=\frac{\left(\delta_{1}^{\prime}+\delta_{2}^{\prime}\right)\left(l_{1} \cos \theta_{1}+l_{2} \cos \theta_{2}\right)}{\left(\delta_{1}+\delta_{2}\right)\left(h+s_{1}+s_{2}+l_{2} \sin \theta_{2}+l_{3} \sin \theta_{3}\right)} .
$$

$$
\begin{aligned}
\varphi^{\prime}= & \frac{\left(\frac{l_{1}}{l_{1}+l_{2}} \cos \theta_{1}\right) l_{1}^{3}}{12 E I} \sin \theta_{1}+\frac{\left(\frac{l_{2}}{l_{1}+l_{2}} \cos \theta_{2}\right) l_{2}^{3}}{12 E I} \sin \theta_{2} \\
& +\frac{\frac{l_{1}}{l_{1}+l_{2}} \sin \theta_{1}}{E \mathrm{bt}} \cos \theta_{1}+\frac{\frac{l_{2}}{l_{1}+l_{2}} \sin \theta_{2}}{E \mathrm{bt}} \cos \theta_{2} .
\end{aligned}
$$

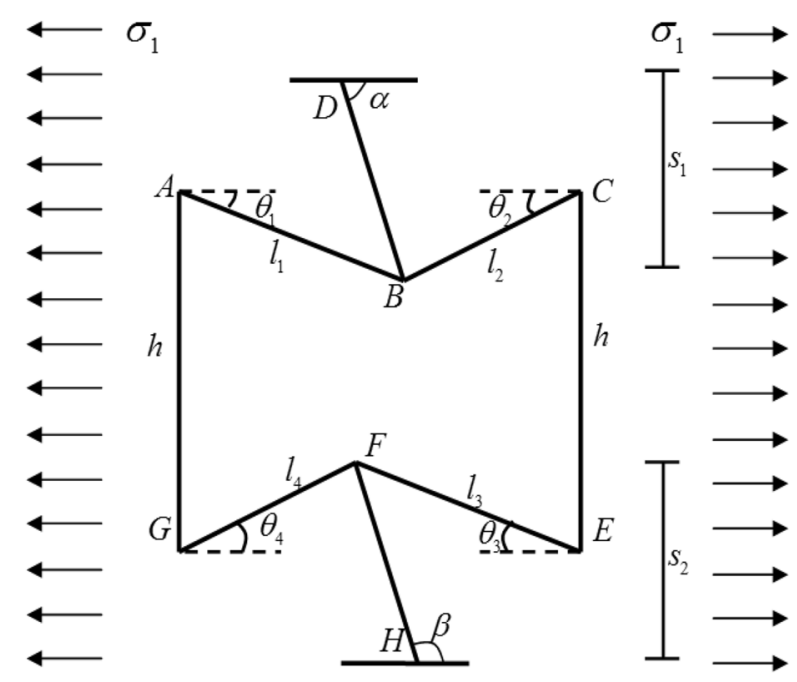

a Stress along the $\sigma_{1}$ direction

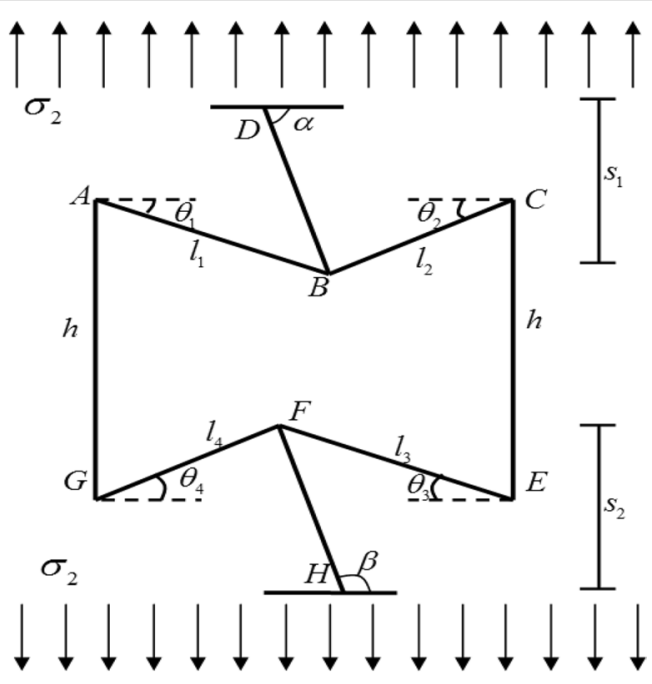

b Stress along the $\sigma_{2}$ direction

Figure $\mathbf{7}$ Tensile stresses along the (a) $\sigma_{1}$ and (b) $\sigma_{2}$ directions 


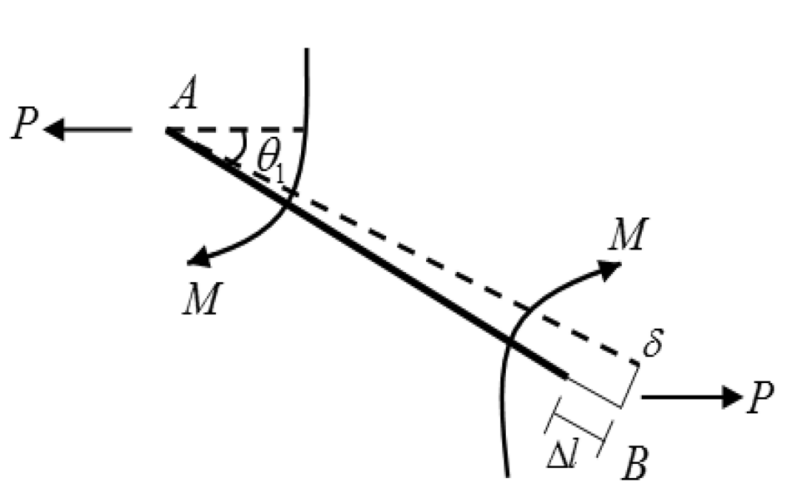

a Force analysis of $\operatorname{rod} A B$

Figure 8 Force analysis of rods $A B$ and GF in the $\sigma_{1}$ and $\sigma_{2}$ directions, respectively

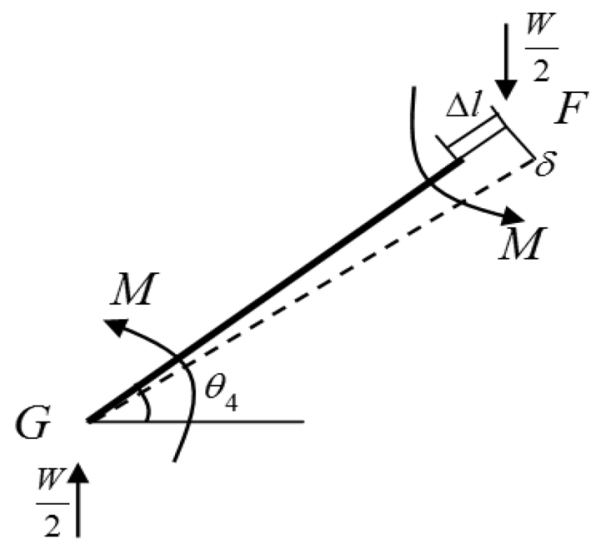

b Force analysis of $\operatorname{rod} G F$
Thus, Poisson's ratio $\nu_{21}$ of a structure in the $\sigma_{2}$ direction can be expressed as

$$
v_{21}=\frac{\varphi^{\prime}\left(h+s_{1}+s_{2}+l_{2} \sin \theta_{2}+l_{3} \sin \theta_{3}\right)}{\varphi\left(l_{1} \cos \theta_{1}+l_{2} \cos \theta_{2}\right)} .
$$

\section{Irregular Honeycomb Structure with Negative Poisson's Ratio}

Figure 7 shows the irregular honeycomb structures loaded in two different directions. $\sigma_{1}$ and $\sigma_{2}$ are uniformly distributed loads in two mutually perpendicular directions.

\subsection{Elastic Modulus in $\sigma_{1}$ Direction}

Figure 8 shows the force analysis of rods $A B$ and $G F$ in the $\sigma_{1}$ and $\sigma_{2}$ directions, respectively. $E_{1}^{\prime}$ is the elastic modulus of an irregular structure with a negative Poisson's ratio and has the same value as an irregular structure with a positive Poisson's ratio. The elastic modulus $E_{1}^{\prime}$ in the $\sigma_{1}$ direction can be obtained as described in Section 3.1:

$$
E_{1}^{\prime}=\frac{\left(l_{1} \cos \theta_{1}+l_{2} \cos \theta_{2}\right)}{\gamma}
$$

where

$$
\begin{aligned}
\gamma= & b\left[\left(h-l_{1} \sin \theta_{1}\right)\left(\frac{l_{1}^{3} \sin ^{2} \theta_{1}}{12 E I}+\frac{\cos ^{2} \theta_{1} l_{1}}{E b t}\right)\right. \\
& \left.+\left(h-l_{2} \sin \theta_{2}\right)\left(\frac{l_{2}^{3} \sin ^{2} \theta_{2}}{12 E I}+\frac{\cos ^{2} \theta_{2} l_{2}}{E b t}\right)\right] .
\end{aligned}
$$

\subsection{Elastic Modulus in the $\sigma_{2}$ Direction}

The elastic modulus of structures with a negative Poisson's ratio can be obtained as described in Section 3.2. Thus, the strain $\varepsilon_{21}$ in the $\sigma_{2}$ direction can be obtained as follows:

$$
\begin{aligned}
\varepsilon_{21} & =\frac{\delta}{h+s_{1}+s_{2}-l_{1} \sin \theta_{1}-l_{4} \sin \theta_{4}} \\
& =\frac{w \varphi}{h+s_{1}+s_{2}-l_{1} \sin \theta_{1}-l_{4} \sin \theta_{4}},
\end{aligned}
$$

where $w=\sigma_{2}\left(l_{1} \cos \theta_{1}+l_{2} \cos \theta_{2}\right) b$.

Thus, the elastic modulus of $E_{2}^{\prime}$ in the $\sigma_{2}$ direction can be expressed as

$$
E_{2}^{\prime}=\frac{\sigma_{21}}{\varepsilon_{21}}=\frac{h+s_{1}+s_{2}-l_{1} \sin \theta_{1}-l_{4} \sin \theta_{4}}{\left(l_{1} \cos \theta_{1}+l_{2} \cos \theta_{2}\right) b \varphi},
$$

where

$$
\begin{aligned}
\varphi= & {\left[\left(\frac{\cos \alpha\left(\frac{s_{1}}{\sin \alpha}\right)^{3}}{3 E I}\right) \cos \alpha+\frac{s_{1} \cot \alpha l_{1}}{l_{1}+l_{2}} \frac{l_{1}}{6 E I}\left(\frac{s_{1}}{\sin \alpha}\right) \cos \alpha\right.} \\
& +\left(\frac{\cos \beta\left(\frac{s_{2}}{\sin \beta}\right)^{3}}{3 E I}\right) \cos \beta+\frac{s_{1} \cot \alpha l_{4}}{l_{3}+l_{4}} \frac{l_{4}}{6 E I}\left(\frac{s_{2}}{\sin \beta}\right) \cos \beta \\
& +\frac{s_{1}}{E b t} \sin \alpha+\frac{s_{2}}{E b t} \sin \beta+\frac{\left(\frac{l_{1}}{l_{1}+l_{2}} \cos \theta_{1}\right) l_{1}^{3}}{12 E I} \cos \theta_{1} \\
& +\frac{\left(\frac{l_{4}}{l_{3}+l_{4}} \cos \theta_{4}\right) l_{4}^{3}}{12 E I} \cos \theta_{4}+\frac{\frac{l_{1}}{l_{1}+l_{2}} \sin \theta_{1} l_{1}}{E b t} \sin \theta_{1} \\
& \left.+\frac{\frac{l_{4}}{l_{3}+l_{4}} \sin \theta_{4} l_{4}}{E b t} \sin \theta_{4}\right] .
\end{aligned}
$$


4.3 Poisson's Ratio $v_{12}^{\prime}$

To derive Poisson's ratio for an irregular structure, the mechanics formula is calculated to obtain Poisson's ratio in the $\sigma_{1}$ direction:

$$
v_{12}^{\prime}=-\frac{\varepsilon_{2}^{\prime \prime}}{\varepsilon_{1}^{\prime \prime}},
$$

where $\varepsilon_{1}^{\prime \prime}$ and $\varepsilon_{2}^{\prime \prime}$ are strains in the $\sigma_{1}$ and $\sigma_{2}$ directions, respectively, due to the load in the $\sigma_{1}$ direction. $v_{12}^{\prime}$ is Poisson's ratio in the $\sigma_{1}$ direction, $\varepsilon_{1}^{\prime \prime}$ can be obtained from Eq. (7), and $\varepsilon_{2}^{\prime \prime}$ can be expressed as

$$
\varepsilon_{2}^{\prime \prime}=\frac{\delta_{1}^{\prime \prime}+\delta_{2}^{\prime \prime}}{h+s_{1}+s_{2}-l_{2} \sin \theta_{2}-l_{3} \sin \theta_{3}},
$$

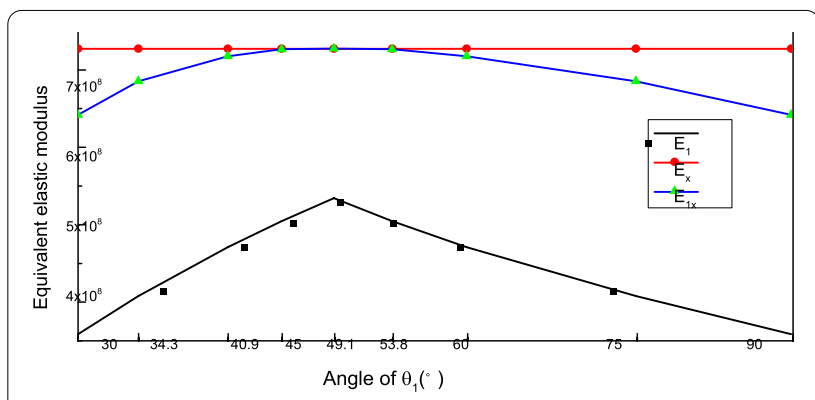

Figure 10 Elastic modulus $E_{1}$ with variation of angle $\theta_{1}$ in the $\sigma_{1}$ direction

where $\delta_{1}^{\prime \prime}$ and $\delta_{2}^{\prime \prime}$ can be expressed as

$$
\left\{\begin{array}{l}
\delta_{1}^{\prime \prime}=\delta_{A B} \cos \theta_{1}+\Delta l \sin \theta_{1}=\frac{p_{1} l_{1}^{3} \sin \theta_{1} \cos \theta_{1}}{12 E I}+\frac{p_{1} l_{1} \sin \theta_{1} \cos \theta_{1}}{E b t} \\
\delta_{2}^{\prime \prime}=\delta_{G F} \cos \theta_{4}+\Delta l \sin \theta_{4}=\frac{p_{2} l_{4}^{3} \sin \theta_{4} \cos \theta_{4}}{12 E I}+\frac{p_{2} l_{4} \sin \theta_{4} \cos \theta_{4}}{E b t}
\end{array}\right.
$$

Thus, Poisson's ratio $v_{12}^{\prime}$ of a structure in the $\sigma_{1}$ direction can be expressed as

$$
v_{12}^{\prime}=-\frac{\left(\delta_{1}^{\prime \prime}+\delta_{2}^{\prime \prime}\right)\left(l_{1} \cos \theta_{1}+l_{2} \cos \theta_{2}\right)}{\left(\delta_{1}+\delta_{2}\right)\left(h+s_{1}+s_{2}-l_{2} \sin \theta_{2}-l_{3} \sin \theta_{3}\right)} .
$$

\subsection{Poisson's Ratio $v_{21}^{\prime}$}

Poisson's ratio $v_{21}^{\prime}$ of a unit structure in the $\sigma_{2}$ direction can be obtained from Section 3.4, as follows:

$$
v_{21}^{\prime}=-\frac{\varphi^{\prime}\left(h+s_{1}+s_{2}-l_{2} \sin \theta_{2}-l_{3} \sin \theta_{3}\right)}{\varphi\left(l_{1} \cos \theta_{1}+l_{2} \cos \theta_{2}\right)},
$$

where

$$
\begin{aligned}
\varphi^{\prime}= & \frac{\left(\frac{l_{1}}{l_{1}+l_{2}} \cos \theta_{1}\right) l_{1}^{3}}{12 E I} \sin \theta_{1}+\frac{\left(\frac{l_{2}}{l_{1}+l_{2}} \cos \theta_{2}\right) l_{2}^{3}}{12 E I} \sin \theta_{2} \\
& +\frac{\frac{l_{1}}{l_{1}+l_{2}} \sin \theta_{1}}{E b t} \cos \theta_{1}+\frac{\frac{l_{2}}{l_{1}+l_{2}} \sin \theta_{2}}{E b t} \cos \theta_{2} .
\end{aligned}
$$

\section{Results and Discussions}

In this study, the geometric configuration of the unit structure with a fixed enclosed area is defined, as shown in Figure 9.

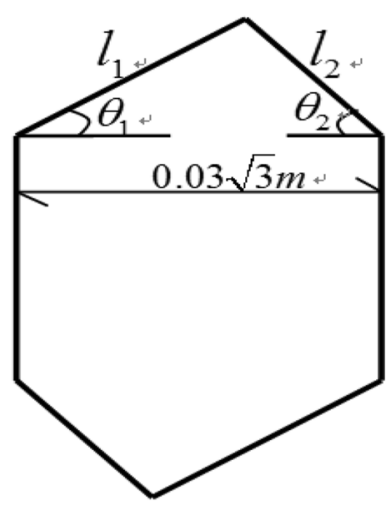

a Positive Poisson's ratio structure

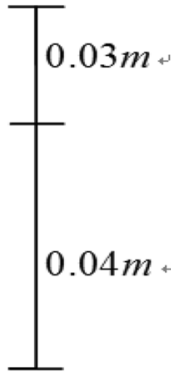

Figure 9 Structure parameter geometry with the same enclosed area

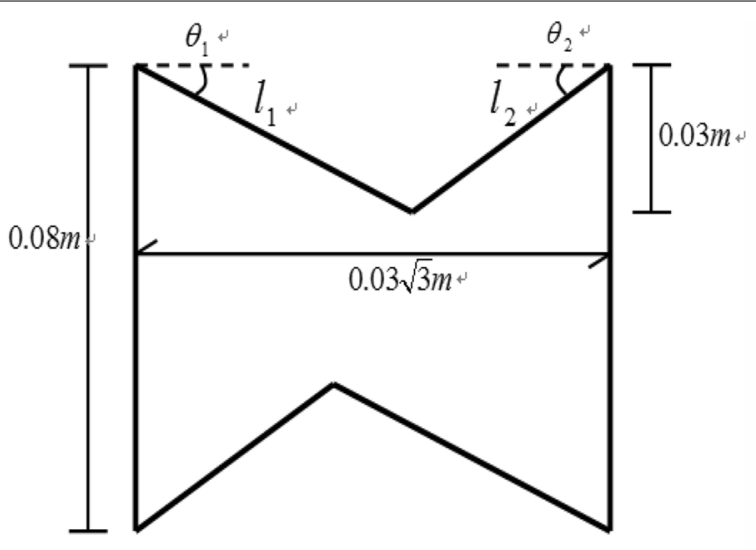

b Negative Poisson's ratio structure 


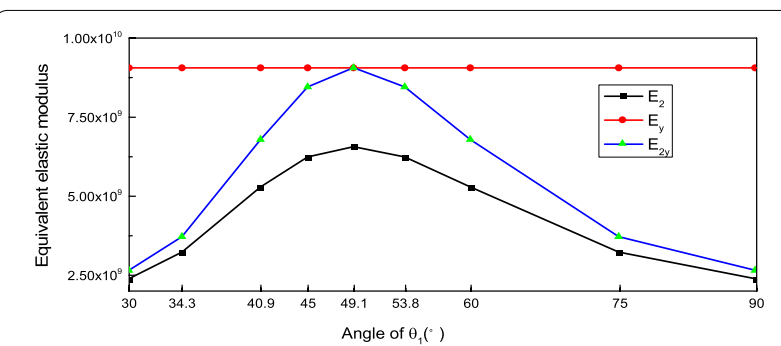

Figure 11 Elastic modulus $E_{2}$ with varying $\theta_{1}$ angle in the $\sigma_{2}$ direction

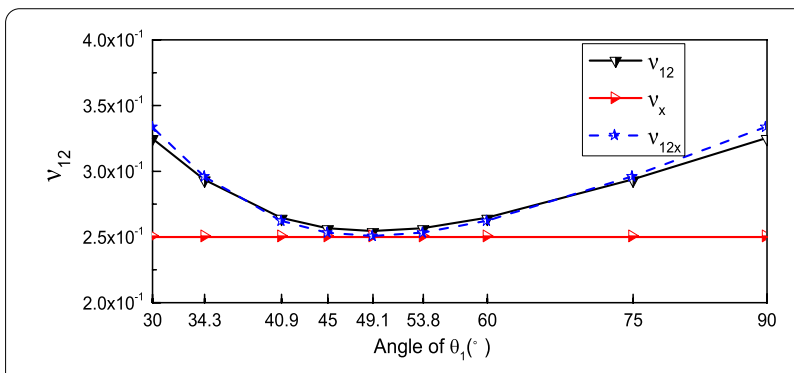

Figure 12 Poisson's ratio $v_{12}$ with varying $\theta_{1}$ angle in the $\sigma_{1}$ direction

Figure 9 shows two structures: a positive and a negative Poisson's ratio structure, which are analyzed with the same enclosed area, where $b=0.01 \mathrm{~m}, t=0.01 \mathrm{~m}$, the original material is aluminum, and the elastic modulus is $70 \times 10^{9} \mathrm{~Pa}$. To obtain the elastic properties, the enclosed area of the structure is fixed in this study, so the variation in $\theta_{1}$ can only influence the structure shape. According to the structure in Figure 9, when the value of $\theta_{1}$ is $30^{\circ}, 34.3^{\circ}, 40.9^{\circ}, 45^{\circ}$, and $49.1^{\circ}, \theta_{2}$ becomes $90^{\circ}$, $75^{\circ}, 60^{\circ}, 53.8^{\circ}$, and $49.1^{\circ}$, respectively, and the irregular structure becomes symmetrical when $\theta_{1}=\theta_{2}=49.1^{\circ}$, which has the same structure as the regular honeycomb structure.

\subsection{Elastic Properties of Structure with Positive Poisson's Ratio}

The equivalent elastic modulus of the positive Poisson's ratio structure due to changes in $\theta_{1}$ is shown in Figure 10, where $E_{1}$ is the equivalent elastic modulus in the $\sigma_{1}$ direction, $E_{x}$ is the equivalent elastic modulus of the regular structure obtained from Ref. [24], and $E_{1 x}$ is the equivalent elastic modulus without considering axial deformation of rods. It can be seen that when $\theta_{1}=\theta_{2}=49.1^{\circ}$ (structure becomes regular), the equivalent elastic modulus reaches the maximum value, and $E_{1 x}$ has the same value as $E_{x}$. Because the structures are identical when $\theta_{1}=\theta_{2}=49.1^{\circ}$, the correctness of the results obtained in this study is verified. When the axial deformation of the rods is considered, the elastic modulus in the $\sigma_{1}$ direction

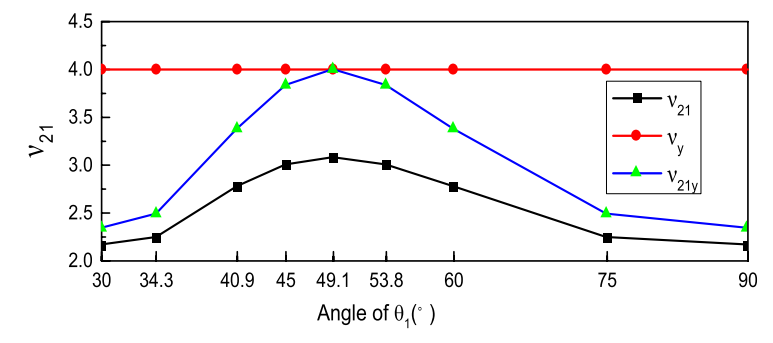

Figure 13 Poisson's ratio with varying $\theta_{1}$ angle in the $\sigma_{2}$ direction

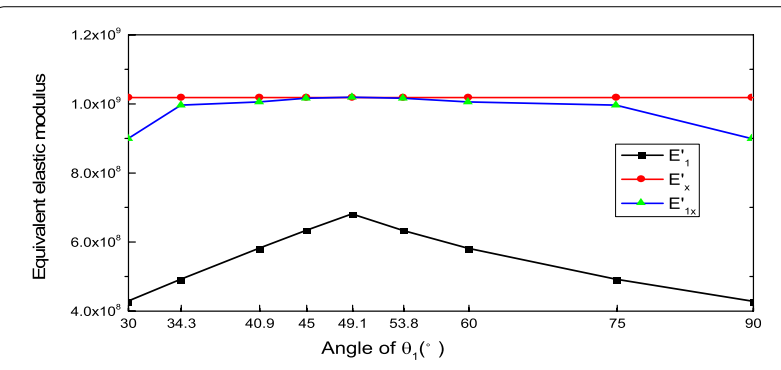

Figure 14 Elastic modulus $E_{1}^{\prime}$ with varying $\theta_{1}$ angle in the $\sigma_{1}$ direction

is lower than that without considering the axial deformation of the rods. When $\theta_{1}$ changes, the values of the elastic modulus of the regular structure are higher than those of the irregular structure.

Figure 11 shows the equivalent elastic modulus of the positive Poisson's ratio structure due to changes in $\theta_{1}$ in the $\sigma_{2}$ direction. $E_{y}$ is the equivalent elastic modulus of the regular structure obtained from Ref. [24]. $E_{2 y}$ is the equivalent elastic modulus calculated without considering the axial deformation in the $\sigma_{2}$ direction. When the $\theta_{1}$ angle is $49.1^{\circ}$ (structure becomes a regular structure), the equivalent elastic modulus reaches the maximum value and $E_{2 y}$ has the same value with $E_{y}$; this proves the validity of the calculation results of this study. With the increase of structural irregularity, differences between $E_{y}$ and $E_{2 y}$ decrease gradually. It can be seen that structure shape and axial deformation have a significant influence on elastic modulus.

Poisson's ratio in the $\sigma_{1}$ direction with variation in $\theta_{1}$ is shown in Figure 12. $v_{x}$ is the Poisson's ratio of the regular structure obtained from Ref. [24]. $v_{12 x}$ is the Poisson's ratio without considering axial deformation in the $\sigma_{1}$ direction. When the $\theta_{1}$ angle is $49.1^{\circ}$ (structure becomes regular), $v_{12 x}$ has the same value as $v_{x}$ and the results obtained from Ref. [24] are the same as the values obtained in this study; this verifies the correctness of the obtained results. It can be seen that when $\theta_{1}$ is lower than $49.1^{\circ}$, Poisson's ratio gradually decreases. Axial deformation has a significant influence on Poisson's ratio, and 


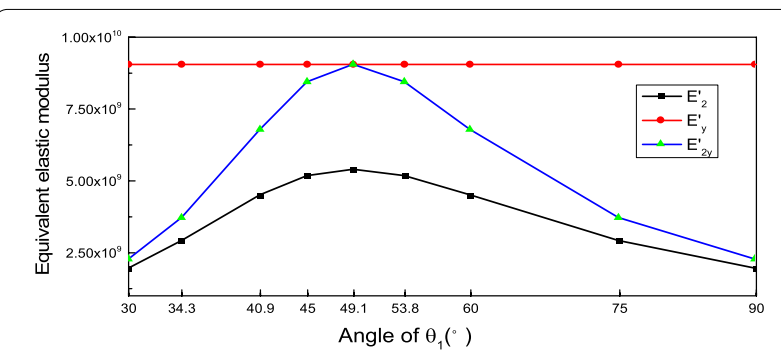

Figure 15 Elastic modulus $E_{2}^{\prime}$ with varying $\theta_{1}$ angle in the $\sigma_{2}$ direction

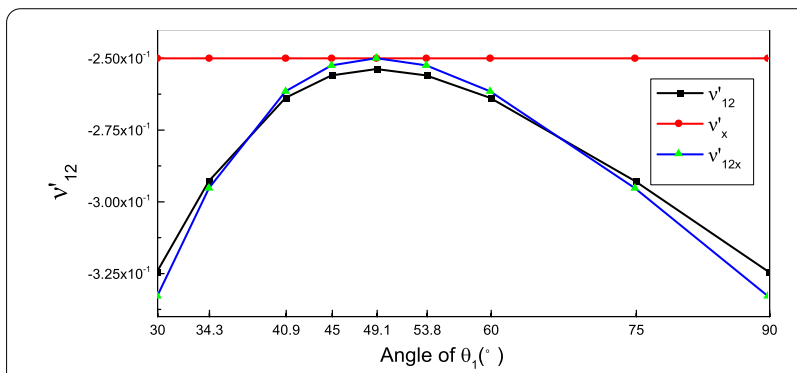

Figure 16 Poisson's ratio with the variation of angle $\theta_{1}$ in $\sigma_{1}$ direction

when the $\theta_{1}$ angle is $49.1^{\circ}$, the Poisson's ratio of the structures reaches the minimum value.

Figure 13 shows the variation of $v_{21}$ with different structural shapes in the $\sigma_{2}$ direction. $v_{y}$ is the Poisson's ratio of the regular structure obtained from Ref. [24]. $v_{21 y}$ is the Poisson's ratio without considering the axial deformation in the $\sigma_{2}$ direction. When the $\theta_{1}$ angle is $49.1^{\circ}$ (structure becomes regular), $v_{21 y}$ and $v_{y}$ have the same value; this verifies the validity of this study. It can be observed that cell angle $\theta_{1}$ influences $\nu_{21}$. Poisson's ratio considering axial deformation is lower than that without considering axial deformation, and positive Poisson's ratio structures reach the maximum value in $49.1^{\circ}$.

\subsection{Elastic Properties of Structure with Negative Poisson's Ratio}

The equivalent elastic modulus of the positive Poisson's ratio structure due to changes in $\theta_{1}$ is shown in Figure 14, where $E_{x}^{\prime}$ is the equivalent elastic modulus of the regular structure obtained from Ref. [24], $E_{1 x}^{\prime}$ is the equivalent elastic modulus of the irregular structure without considering axial deformation, and $E_{1}^{\prime}$ is the equivalent elastic modulus of an irregular structure considering axial deformation. It can be seen that $E_{1 x}^{\prime}$ and $E_{x}^{\prime}$ have the same value when $\theta_{1}=\theta_{2}=49.1^{\circ}$, which verifies the correctness of the results in this study, and the equivalent elastic modulus of the structures reaches the maximum value at $\theta_{1}=49.1^{\circ}$. When the $\theta_{1}$ angle changes, the elastic modulus decreases with an increase in structural irregularity.

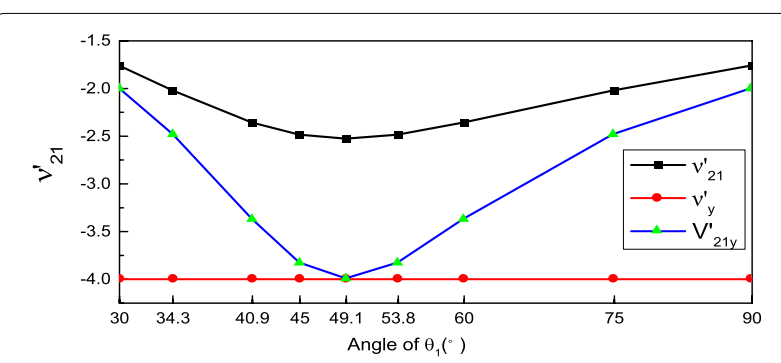

Figure 17 Poisson's ratio with varying $\theta_{1}$ angle in the $\sigma_{2}$ direction

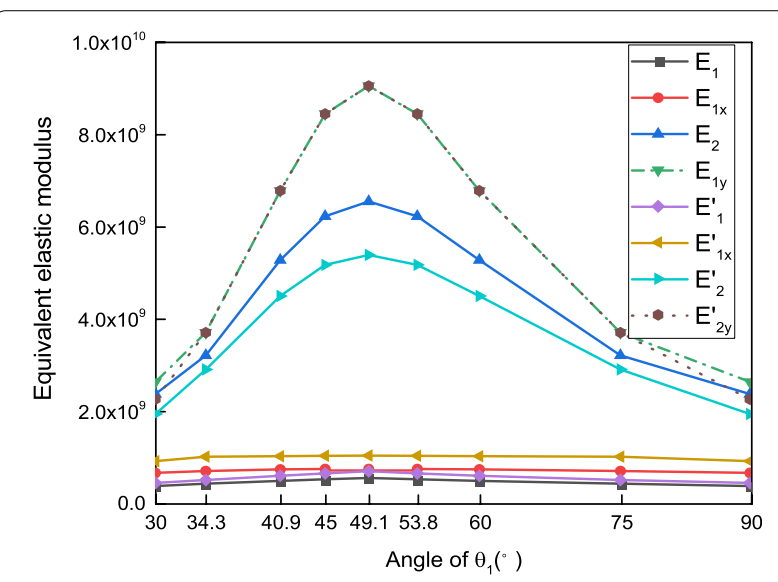

Figure 18 Equivalent elastic modulus of two structures in the $\sigma_{1}$ and $\sigma_{2}$ directions

Figure 15 shows the equivalent elastic modulus of the negative Poisson's ratio structure due to changes in $\theta_{1}$ in the $\sigma_{2}$ direction. $E_{y}^{\prime}$ is the equivalent elastic modulus of the regular structure obtained from Ref. [24]. $E_{2 y}^{\prime}$ is the equivalent elastic modulus calculated without considering axial deformation in the $\sigma_{2}$ direction. When the $\theta_{1}$ angle is $49.1^{\circ}$, the equivalent elastic modulus reaches the maximum value and $E_{2 y}^{\prime}$ is the same value with the result reported in Ref. [24]; this verifies the correctness of the results obtained in this study. The elastic modulus considering axial deformation is lower than that without considering axial deformation, and the difference between them reaches the maximum value when $\theta_{1}=$ $49.1^{\circ}$. Whether or not the axial deformation of the rod is considered has a great influence on the equivalent elastic modulus.

The Poisson's ratio of the negative Poisson's ratio structure in the $\sigma_{1}$ direction is shown in Figure 16. $v_{x}^{\prime}$ is the Poisson's ratio of the regular structure obtained from Ref. [24]. $v_{12 x}^{\prime}$ is the Poisson's ratio without considering the axial deformation in the $\sigma_{1}$ direction. $v_{12}^{\prime}$ is the Poisson's ratio considering the axial deformation in the $\sigma_{1}$ direction. When $\theta_{1}=\theta_{2}=49.1^{\circ}$, the Poisson's ratios of the structure reaches the maximum value and $v_{12 x}^{\prime}$ has the 
same value as $v_{x}^{\prime}$, verifying the correctness of the results obtained in this study. The consideration of axial deformation has a negligible influence on Poisson's ratio in the $\sigma_{1}$ direction. When the $\theta_{1}$ angle is lower than $49.1^{\circ}, v_{12 x}^{\prime}$ increases with an increase in the $\theta_{1}$ angle, and when $\theta_{1}$ is higher than $49.1^{\circ}, v_{12 x}^{\prime}$ decreases with an increase in the $\theta_{1}$ angle.

Figure 17 shows the variation in $v_{21}$ with different structural shapes in the $\sigma_{2}$ direction. $v_{y}^{\prime}$ is the Poisson's ratio of the regular structure obtained from Ref. [24]. $v_{12 y}^{\prime}$ is the Poisson's ratio without considering the axial deformation in the $\sigma_{2}$ direction. When the cell angle $\theta_{1}$ is $49.1^{\circ}$, $v_{21}^{\prime}$ and $v_{12 y}^{\prime}$ reach their minimum values. The value of $v_{12 y}^{\prime}$ without considering axial deformation is the same as the results of Ref. [24] at an angle of $49.1^{\circ}$. Figure 17 also shows that the Poisson's ratio considering axial deformation is higher than that without considering axial deformation, and the difference between them reaches the maximum value when $\theta_{1}=49.1^{\circ}$.

Figure 18 shows the equivalent elastic moduli of the irregular structures in the $\sigma_{1}$ and $\sigma_{2}$ directions. $E_{1}, E_{1 x}$, $E_{2}, E_{1 y}, E_{1}^{\prime}, E_{1 x}^{\prime}, E_{2}^{\prime}$ and $E_{2 y}^{\prime}$ are the equivalent elastic moduli obtained from Figures 10, 11, 12, 13, 14, 15, 16 and 17. It can be seen that the equivalent elastic moduli in the $\sigma_{2}$ direction is higher than those in the $\sigma_{1}$ direction. When $\theta_{1}$ $=\theta_{2}=49.1^{\circ}$, the equivalent elastic moduli in the $\sigma_{2}$ direction reach the maximum value, and $E_{2 y}^{\prime}$ is the same value with $E_{1 y}$. The axial deformation of rods has a significant influence on the equivalent elastic moduli. The equivalent elastic moduli in the $\sigma_{1}$ direction have insignificant variation when $\theta_{1}$ varies.

Figure 19 shows the Poisson's ratio of the irregular structures in the $\sigma_{1}$ and $\sigma_{2}$ directions. $v_{12}, v_{12 x}, v_{21}, v_{21 y}$, $v_{12}^{\prime}, v_{12 x}^{\prime}, v_{21}^{\prime}$, and $v_{21 y}^{\prime}$ are the equivalent elastic moduli obtained from Figures 10, 11, 12, 13, 14, 15, 16 and 17. Notably, the value of $v_{21 y}$ is higher than the rest. The

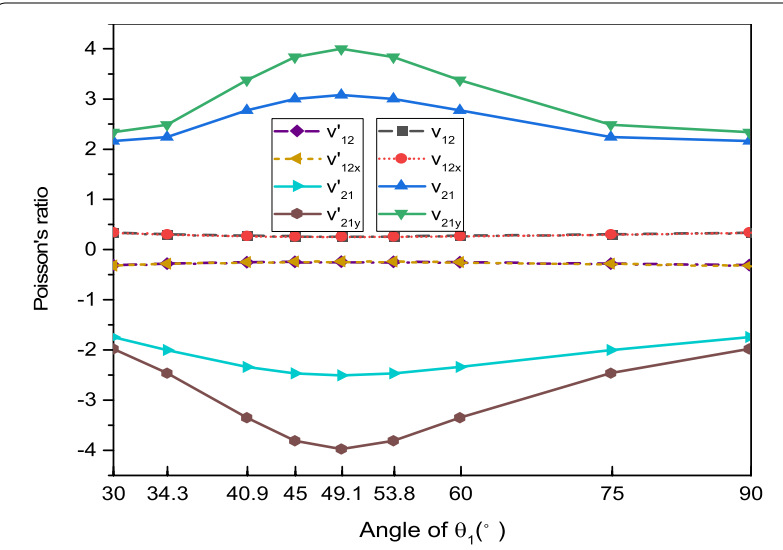

Figure 19 Poisson's of two structures in the $\sigma_{1}$ and $\sigma_{2}$ directions absolute value of the Poisson's ratio in two structures reaches the maximum value. Moreover, $v_{12}, v_{12 x}$ and $v_{12}^{\prime}$, $v_{12 x}^{\prime}$ have similar values, respectively. Whether or not the axial deformation is considered has little influence on the Poisson's ratio of the structure.

\section{Conclusions}

In this study, two types of irregular honeycomb structures were studied using material mechanics and structural mechanics methods. Considering axial deformation of rods, the elastic properties of irregular structures with different structure shapes were studied. Compared with the results reported in Ref. [24], the results were in good agreement.

The results show that when the enclosed area of the irregular honeycomb structure is fixed, the equivalent elastic modulus and Poisson's ratio of the structure will vary with varying structure shape. The $\theta_{1}$ angle has a significant influence on the equivalent elastic modulus. When the structure is regular, the absolute value of the elastic modulus and Poisson's ratio in the $\sigma_{2}$ direction reaches the maximum value, and the Poisson's ratio in the $\sigma_{1}$ direction reaches the minimum value. The elastic properties of the structure considering axial deformation are higher than those without considering the axial deformation. Therefore, the elastic properties of irregular structures can be achieved by the axial deformation of the rods, structural shapes, and original materials.

\section{Acknowledgements \\ Not applicable.}

\section{Authors' contributions}

NW and QD were in charge of the whole analysis; NW and QD wrote the manuscript. All authors read and approved the final manuscript.

\section{Authors' Information}

Ning Wang, born in 1995, is currently MD candidate at School of Science, Chang'an University, China. His research interests include cellular structures.

Qingtian Deng, born in 1980, is currently an associate professor at Chang'an University, China. He received his PhD degree on Solid Mechanics from Hunan University, China, in 2008.

\section{Funding}

Supported by Fundamental Research Funds for the Central Universities (Grant No. 310812161003) and Natural Science Basic Research Plan in Shaanxi Province of China (Grant No. 2016JM5035).

\section{Competing Interests}

The authors declare no competing financial interests.

Received: 15 February 2020 Revised: 7 April 2021 Accepted: 19 May 2021 Published online: 01 June 2021 


\section{References}

[1] D H Chen, L Yang. Analysis of equivalent elastic modulus of asymmetrical honeycomb. Composite Structures, 2011, 93(2): 767-773.

[2] X Huang, S Blackburn. Developing a new processing route to manufacture honeycomb ceramics with negative Poisson's ratio. Key Engineering Materials, 2001, 206: 201-204.

[3] J J Zhang, G X Liu, Z You. Large deformation and energy absorption of additively manufactured auxetic materials and structures: A review. Composite Part B: Engineering, 2020, 201(15): 108340.

[4] D X Qing, Q Y Liu, CW He, et al. Impact energy absorption of functionally graded chiral honeycomb structures. Extreme Mechanics Letters, 2020, 32: 568-568.

[5] JW Xiang, J X Du. Energy absorption characteristics of bi-inspired honeycomb structure under axial impact loading. Materials Science and Engineering: A, 2017, 696(1): 283-289.

[6] L L Yan, KY Zhu, N Chen, et al. Energy-absorption characteristics of tube-reinforced absorbent honeycomb sandwich structure. Composite Structure, 2021, 255(1): 112946.

[7] M Dhaheri, K A Khan, R Umer, et al. Process-induced deformation in U-shaped honeycomb aerospace composite structures. Composite Structure, 2020, 248(15): 112503.

[8] O A Ganilova, M P Cartmell, A Kiley. Experimental investigation of the thermoelastic performance of an aerospace aluminium honeycomb composite panel. Composite Structure, 2021, 257(1): 113159.

[9] Z D Ma, H Bian, C Sun, et al. Functionally-grand NPR(Negative Poisson's Ratio) material for a blast-protective deflector. Dearborn, Michigan: DTIC Document, 2010: 1109.

[10] D Q Yang, T Ma, G L Zhang. A new honeycomb side protection structure with macroscopically negative Poisson's ratio effect. Explosion and Shock Waves, 2015, 25(2): 243-248. (in Chinese)

[11] D H Zhang, Q G Fei, J Z Liu, et al. Crushing of vertex-based hierarchical honeycombs with triangular substructures. Thin-Walled Structures, 2020, 146: 106436.

[12] T Mukhopadhyay, S Adhikari. Effective in-plane elastic properties of auxetic honeycombs with spatial irregularity. Mechanics of Materials, 2016, 95 204-222.

[13] Z C Yang, Q T Deng. Research and application of mechanical properties in materials and structures with negative Poisson's ratio. Advances in Mechanics, 2011, 41(3): 87-102. (in Chinese)

[14] L H Lan, J Sun, F L Hong, et al. Nonlinear constitutive relations of thinwalled honeycomb structure. Mechanics of Materials, 2020, 149: 103556.

[15] S Upreti, V K Singh, S K Karmal, et al. Modelling and analysis of honeycomb sandwich structure using finite element method. Materials: Proceedings, 2020, 25(4): 620-625.

[16] Y H Wang, JY R Liew, S C Lee. Experimental and numerical studies of non-composite Steel-Concrete-Steel sandwich panels under impulsive loading. Materials \& Design, 2015, 5(33): 104-112.

[17] Z Li, T Wang, Y Jiang, L M Wang, et al. Design-oriented crushing analysis of hexagonal honeycomb core under in-plane compression. Composite Structures, 2017, 12(66): 429-438.

[18] D Asprone, F Auricchio, C Menna, et al. Statistical finite element analysis of the buckling behavior of honeycomb structures. Composite Structures, 2013, 105: 240-255.
[19] D Okumura, N Ohno, H Noguchi. Post-buckling analysis of elastic honeycombs subject to in-plane biaxial compression. International Journal of Solids and Structures, 2002, 39(13): 3487-3503.

[20] K Wei, Y Peng, W B Wen, et al. Tailorable thermal expansion of lightweight and robust dual-constituent triangular lattice material. Journal of Applied Mechanics-Transactions of ASME, 2017, 84(10): 101006

[21] X L Wang, D Y Bai. Thermal expansion and thermal fluctuation effects in a binary granular mixture. International Journal of Heat and Mass Transfer, 2018, 116: 84-92.

[22] J Zhang, H Zhai, Z H Wu, et al. Enhanced performance of photovoltaic thermoelectric coupling devices with thermal interface materials. Energy Reports, 2020, 6: 116-122.

[23] HXu, A Farag, D Pasini. Multilevel hierarchy in bi-material lattices with high specific stiffness and unbounded thermal expansion. Acta Materialia, 2017, 134(1): 155-166.

[24] T Mukhopadhyay, S Adhikari. Equivalent in-plane elastic properties of irregular honeycombs: An analytical approach. International Journal of Solids and Structures, 2016, 91: 169-184

[25] C Li, H S Hui, H Wang. Nonlinear bending of sandwich beams with functionally graded negative Poisson's ratio honeycomb core. Composite Structure, 2019, 134(79): 1140.

[26] QT Deng, Z C Yang. Effects of Poisson's ratio on functionally graded cellular structure. Material Express, 2016, 6(6).

[27] L L Hu, Z R Luo, Q Y Yin. Negative Poisson's ratio effect of re-entrant antitrichiral honeycombs under large deformation. Thin-Walled Structures, 2019, 141: 283-292.

[28] J Huang, X B Gong, Q H Zhang, et al. In-plane mechanics of a novel zero Poisson's ratio honeycomb core. Composite Part B: Engineering, 2016, 89(15): 67-76

[29] TWang, L M Wang, Z D Ma, et al. Elastic analysis of auxetic cellular structure consisting of re-entrant hexagonal cells using a strain-based expansion homogenization method. Materials and Design, 2018, 160(15): 284-293.

[30] E A Bubert, B K S Woods, K Lee, et al. Design and fabrication of a passive 1D morphing aircraft skin. Journal of Intelligent Material Systems and Structures, 2010, 21(17): 1699-1717.

[31] K Li, X L Gao, J Wang. Dynamic crushing behavior of honeycomb structures with irregular cell shapes and non-uniform cell wall thickness. International Journal of Solids and Structures, 2007, 44(14): 5003-5026.

[32] J F Liu, W S Chen, H Hao, et al. In-plane crushing behaviors of hexagonal honeycombs with different Poisson's ratio induced by topological diversity. Thin-Walled Structures, 2021, 159: 107223.

[33] R J Roark. Formulas for stress and strain. Journal of Applied Mechanics, 1989, 43(3): 624

[34] WY Liu, N L Wang, J L Huang, et al. The effect of irregularity, residual convex units and stresses on the effective mechanical properties of 2D auxetic cellular structure. Materials Science and Engineering: A, 2014, 609(15): 26-33.

\section{Submit your manuscript to a SpringerOpen ${ }^{\oplus}$ journal and benefit from:}

- Convenient online submission

- Rigorous peer review

- Open access: articles freely available online

- High visibility within the field

Retaining the copyright to your article

Submit your next manuscript at springeropen.com 